\title{
Numerical Method Using Cubic Trigonometric B-Spline Technique for Nonclassical Diffusion Problems
}

\author{
Muhammad Abbas, ${ }^{1,2}$ Ahmad Abd. Majid, \\ Ahmad Izani Md. Ismail, ${ }^{2}$ and Abdur Rashid ${ }^{3}$ \\ ${ }^{1}$ Department of Mathematics, University of Sargodha, Sargodha 40100, Pakistan \\ ${ }^{2}$ School of Mathematical Sciences, Universiti Sains Malaysia (USM), 11800 Penang, Malaysia \\ ${ }^{3}$ Department of Mathematics, Gomal University, Dera Ismail Khan 29050, Pakistan
}

Correspondence should be addressed to Muhammad Abbas; m.abbas@uos.edu.pk

Received 17 October 2013; Accepted 13 April 2014; Published 20 May 2014

Academic Editor: Sining Zheng

Copyright ( 2014 Muhammad Abbas et al. This is an open access article distributed under the Creative Commons Attribution License, which permits unrestricted use, distribution, and reproduction in any medium, provided the original work is properly cited.

A new two-time level implicit technique based on cubic trigonometric B-spline is proposed for the approximate solution of a nonclassical diffusion problem with nonlocal boundary constraints. The standard finite difference approach is applied to discretize the time derivative while cubic trigonometric B-spline is utilized as an interpolating function in the space dimension. The technique is shown to be unconditionally stable using the von Neumann method. Several numerical examples are discussed to exhibit the feasibility and capability of the technique. The $L_{2}$ and $L_{\infty}$ error norms are also computed at different times for different space size steps to assess the performance of the proposed technique. The technique requires smaller computational time than several other methods and the numerical results are found to be in good agreement with known solutions and with existing schemes in the literature.

\section{Introduction}

This study deals with the numerical solution of a nonclassical diffusion problem with two nonlocal boundary constraints using cubic trigonometric B-splines. This problem arises in several branches of science. In particular, electrochemistry [1], heat conduction process [2], thermoelasticity [3], plasma physics [4], semiconductor modeling [5], biotechnology [6], control theory, and inverse problems [7]. The analysis, development, and implementation of numerical methods for the solution of such diffusion problems have received wide attention in the literature.

Consider an insulated rod of length $L$ located on the $x$ axis of the interval $[0, L]$. Let the rod have a source of heat. Let $u(x, t)$ denote the temperature in the insulated rod with ends held at constant temperatures $T_{1}$ and $T_{2}$, and the initial temperature distribution along the rod is $g_{1}(x)$. The problem is to study the flow of heat in the rod and in this paper the partial differential equation governing the flow of heat in the rod is given by the diffusion equation with specification of energy:

$$
\frac{\partial u}{\partial t}(x, t)=\alpha^{2} \frac{\partial^{2} u}{\partial x^{2}}(x, t)+q(x, t) \quad 0 \leq x \leq L, 0 \leq t \leq T
$$

with the initial constraint

$$
u(x, t=0)=g_{1}(x) \quad 0 \leq x \leq L
$$

and the nonlocal boundary constraints

$$
\begin{aligned}
\xi_{1} u(x & =0, t)+\xi_{2} u_{x}(x=0, t) \\
& =\int_{0}^{L} g_{2}(x) u(x, t) d x+h_{1}(t)=T_{1}, \\
\xi_{3} u(x & =L, t)+\xi_{4} u_{x}(x=L, t)
\end{aligned}
$$




$$
\begin{array}{r}
\int_{0}^{L} g_{3}(x) u(x, t) d x+h_{2}(t)=T_{2} \\
0<t \leq T,
\end{array}
$$

where $\xi_{i}, i=1,2,3,4$ are known constants, $q, g_{i}(i=1,2,3)$ are known continuous functions, $\alpha$ is the thermal diffusivity of the rod, and $h_{i}(i=1,2)$ are prescribed functions. This problem has been studied by Dehghan [8], Martín-Vaquero and Vigo-Aguiar [9], Li and $\mathrm{Wu}$ [10], and Golbabai and Javidi [11]. Several physical circumstances might be modeled by equation and constraints (1)-(3) and several examples of application in physics with comprehensive derivations of the above mentioned problem can be found in [3, 12-14].

There are several numerical methods in the literature that have been developed for solving the proposed problem (1) subject to initial and nonlocal boundary constraints (2)(3). The methods were based, for instance, on the forward Euler method, the backward Euler approach or the Crank-Nicolson scheme [15, 16], Laplace transformation [17], and so forth. Dehghan [8] presented four finite difference approaches, namely, the BTCS (backward time centred space) scheme, the implicit $(3,3)$ Crandall's formula, the 3-point FTCS (forward time centred space) two-level scheme, and the Dufort-Frankel three-level approach for the numerical solution of parabolic equation with nonlocal specification. Martín-Vaquero and Vigo-Aguiar [9] improved the order of convergence of the implicit $(3,3)$ Crandall's formula proposed by Dehghan [8] and also improved the accuracy of the method. Martín-Vaquero and Vigo-Aguiar [18] developed an algorithm for the solution of the heat conduction equations with nonlocal constraints which reduced the CPU time and enhanced the accuracy of $(3,3)$ Crandall's formula proposed in [8]. $\mathrm{Li}$ and $\mathrm{Wu}[10]$ proposed an algorithm which was based on the transverse method of lines (TMOL) which can reduce a nonclassical diffusion equation to a series of ordinary differential equations (ODEs). Subsequently, the authors in [10] used an analytic reproducing kernel technique to solve ODEs with integral boundary constraints. Dehghan $[19,20]$, jointly with Tatari and Dehghan [21], has proposed several efficient techniques for the numerical solutions of partial differential equations subject to nonlocal boundary constraints. Golbabai and Javidi [11] introduced a Chebyshev spectral collocation method (CSCM) based on Chebyshev polynomials for solving a parabolic problem subject to nonlocal boundary constraints. For more details on other numerical methods for the solution of a one-dimensional heat equation subject to nonlocal boundary constraints in the literature, see [22-31].

The study of B-spline functions is a key element in computer-aided geometric design [32-35]. It has also attracted attention in the literature [36-51] to the numerical solution of various differential equations [38-40]. This is because they have important geometric properties and features that make them amenable to more detailed analysis. Numerical methods based on B-spline functions of various degrees have been utilized for solving initial and boundary value problems. As examples, a cubic B-spline collocation method was used to solve a nonlinear diffusion equation subject to certain initial and Dirichlet boundary constraints [41], a finite element method based on bivariate splines has been used for solving parabolic partial differential equation [42], and the combination of finite difference approach and cubic B-spline method was applied for the solution of a one-dimensional heat equation subject to local boundary constraints [43, 44]. Goh et al. [45] presented a comparison of cubic B-spline and extended cubic uniform B-spline based collocation methods for solving a one-dimensional heat equation with a nonlocal initial constraint and concluded that extended cubic uniform B-spline with an appropriate value of parameters gives better results than the cubic B-spline. A finite difference scheme based on cubic B-spline was also used for solving the one-dimensional wave equation [43], advection-diffusion equation [44], one-dimensional coupled viscous Burgers' equation [47], system of strongly coupled reaction-diffusion equations [48], and one-dimensional hyperbolic problems [49].

In our present paper, a new two-time level implicit technique is developed to approximate the solution of the nonclassical diffusion problem (1) subject to initial constraints in (2) and nonlocal boundary constraints in (1)(3). The technique is based on the cubic trigonometric Bspline functions. A finite difference approach and $\theta$-weighted scheme are applied for the time and space discretization, respectively. Some researchers have considered the ordinary B-spline collocation method for solving the heat equation subject to local and nonlocal boundary constraints but, so far as we are aware, not with the cubic trigonometric Bspline collocation method. Cubic trigonometric B-spline is used as an interpolating function in the space dimension. The unconditional stability property of the method is proved by von Neumann method. The feasibility of the method is shown by test problems with $k=s h^{2}, s=1,2,4,5$ instead of smaller time step size $k=0.4 h^{2}$ and the approximated solutions are found to be in good agreement with the known exact solutions.

The outline of this study is as follows. A numerical solution of nonclassical diffusion problem is presented in Section 2. In Section 3, the cubic trigonometric B-spline is utilized as an interpolating function in the space dimension. The von Neumann approach is used to prove the stability of the method in Section 4. Numerical examples are considered in Section 5 to show the achievability of the proposed method. Finally, the concluding remarks of this study are given in Section 6.

\section{Solution of Nonclassical Diffusion Problem}

Consider a uniform mesh $\Omega$ with grid points $\left(x_{i}, t_{n}\right)$ to discretize the grid region $\Delta=[a, b] \times[0, T]$ with $x_{i}=a+i h$, $i=0,1,2, \ldots, N$ and $t_{n}=n k, n=0,1,2,3, \ldots, M, M k=T$. Here the quantities $h$ and $k$ are mesh space size and time step size, respectively. The time derivative can be approximated by using the standard finite difference formula:

$$
\frac{\partial u^{n}}{\partial t}=\frac{u^{n+1}-u^{n}}{k} .
$$


Using the approximation of (4), (1) becomes

$$
\frac{u^{n+1}-u^{n}}{k}=\alpha^{2} \frac{\partial^{2} u^{n}}{\partial x^{2}}+q\left(x_{i}, t_{n+1}\right) .
$$

Using $\theta$-weighted technique, the space derivatives of (5) can be written as

$$
\begin{aligned}
\frac{u^{n+1}-u^{n}}{k}= & \theta\left(\alpha^{2} \frac{\partial^{2} u^{n+1}}{\partial x^{2}}\right)+(1-\theta)\left(\alpha^{2} \frac{\partial^{2} u^{n}}{\partial x^{2}}\right) \\
& +q\left(x_{i}, t_{n+1}\right),
\end{aligned}
$$

where $0 \leq \theta \leq 1$ and the subscripts $n$ and $n+1$ are successive time levels. It is noted that the system becomes an explicit scheme when $\theta=0$, a fully implicit scheme when $\theta=1$, and a Crank-Nicolson scheme when $\theta=1 / 2[43,49]$. In this paper, we use the Crank-Nicolson approach. Hence, (6) becomes

$$
\frac{u^{n+1}-u^{n}}{k}=\frac{1}{2}\left(\alpha^{2} \frac{\partial^{2} u^{n+1}}{\partial x^{2}}\right)+\frac{1}{2}\left(\alpha^{2} \frac{\partial^{2} u^{n}}{\partial x^{2}}\right)+q\left(x_{i}, t_{n+1}\right) \text {. }
$$

After simplification, (7) leads to

$$
2 u^{n+1}-k \alpha^{2} u_{x x}^{n+1}=2 u^{n}+k \alpha^{2} u_{x x}^{n}+2 k q\left(x_{i}, t_{n+1}\right) .
$$

The space derivatives are approximated by using cubic trigonometric B-spline and are discussed in the next section.

\section{Cubic Trigonometric B-Spline Technique}

In this section, we discuss the cubic trigonometric B-spline collocation method (CuTBSM) for the numerical solution of the nonclassical diffusion equation (1). Consider a mesh $a \leq$ $x \leq b$ which is equally divided by knots $x_{i}$ into $N$ subintervals $\left[x_{i}, x_{i+1}\right], i=0,1,2, \ldots, N-1$ where $a=x_{0}<x_{1}<\cdots<$ $x_{N}=b$. Our approach for the nonclassical diffusion equation using collocation method with cubic trigonometric B-spline is to seek an approximate solution as [37]

$$
U(x, t)=\sum_{i=-3}^{N-1} C_{i}(t) T B_{i}(x),
$$

where $C_{i}(t)$ are to be determined for the approximated solutions $U(x, t)$ to the exact solutions $u(x, t)$, at the point $\left(x_{i}, t_{n}\right) . T B_{i}(x)$ are twice continuously differentiable piecewise cubic trigonometric B-spline basis functions over the mesh defined by [49-51]

$$
\begin{aligned}
& T B_{i}(x) \\
& \quad=\frac{1}{\omega}\left\{\begin{array}{cc}
p^{3}\left(x_{i}\right), & x \in\left[x_{i}, x_{i+1}\right] \\
p\left(x_{i}\right)\left(p\left(x_{i}\right) q\left(x_{i+2}\right)\right. & \\
\left.+q\left(x_{i+3}\right) p\left(x_{i+1}\right)\right) & \\
+q\left(x_{i+4}\right) p^{2}\left(x_{i+1}\right), & x \in\left[x_{i+1}, x_{i+2}\right] \\
q\left(x_{i+4}\right)\left(p\left(x_{i+1}\right) q\left(x_{i+3}\right)\right. & \\
\left.+q\left(x_{i+4}\right) p\left(x_{i+2}\right)\right) & \\
+p\left(x_{i}\right) q^{2}\left(x_{i+3}\right), & x \in\left[x_{i+2}, x_{i+3}\right] \\
q^{3}\left(x_{i+4}\right), & x \in\left[x_{i+3}, x_{i+4}\right],
\end{array}\right.
\end{aligned}
$$

TABLE 1: Values $\operatorname{TB}_{i}(x)$ and its derivatives.

\begin{tabular}{lccccc}
\hline$x$ & $x_{i}$ & $x_{i+1}$ & $x_{i+2}$ & $x_{i+3}$ & $x_{i+4}$ \\
\hline $\mathrm{TB}_{i}$ & 0 & $a_{1}$ & $a_{2}$ & $a_{1}$ & 0 \\
$\mathrm{~TB}_{i}^{\prime}$ & 0 & $a_{3}$ & 0 & $a_{4}$ & 0 \\
$\mathrm{~TB}_{i}^{\prime \prime}$ & 0 & $a_{5}$ & $a_{6}$ & $a_{5}$ & 0 \\
\hline
\end{tabular}

where

$$
\begin{gathered}
p\left(x_{i}\right)=\sin \left(\frac{x-x_{i}}{2}\right), \quad q\left(x_{i}\right)=\sin \left(\frac{x_{i}-x}{2}\right) \\
\omega=\sin \left(\frac{h}{2}\right) \sin (h) \sin \left(\frac{3 h}{2}\right)
\end{gathered}
$$

and where $h=(b-a) / n$. The approximations $U_{i}^{n}$ at the point $\left(x_{i}, t_{n}\right)$ over subinterval $\left[x_{i}, x_{i+1}\right]$ can be defined as

$$
U_{i}^{n}=\sum_{j=i-3}^{i-1} C_{k}^{n} T B_{j}(x)
$$

In order to obtain the approximations to the solutions, the values of $T B_{i}(x)$ and its derivatives at nodal points are required and these derivatives are tabulated in Table 1, where

$$
\begin{aligned}
& a_{1}=\frac{\sin ^{2}(h / 2)}{\sin (h) \sin (3 h / 2)}, \\
& a_{2}=\frac{2}{1+2 \cos (h)}, \\
& a_{3}=-\frac{3}{4 \sin (3 h / 2)}, \\
& a_{4}=\frac{3}{4 \sin (3 h / 2)}, \\
& a_{5}=\frac{3(1+3 \cos (h))}{16 \sin ^{2}(h / 2)(2 \cos (h / 2)+\cos (3 h / 2))}, \\
& a_{6}=-\frac{3 \cos ^{2}(h / 2)}{\sin ^{2}(h / 2)(2+4 \cos (h))} .
\end{aligned}
$$

Using approximate functions (10) and (12) and following Mittal and Arora [46], the values at the knots of $U_{i}^{n}$ and their derivatives up to second order are determined in terms of time parameters $C_{j}^{n}$ as

$$
\begin{gathered}
u_{i}^{n}=a_{1} C_{i-3}^{n}+a_{2} C_{i-2}^{n}+a_{1} C_{i-1}^{n}, \\
\left(u_{x}\right)_{i}^{n}=a_{3} C_{i-3}^{n}+a_{4} C_{i-1}^{n},
\end{gathered}
$$$$
\left(u_{x x}\right)_{i}^{n}=a_{5} C_{i-3}^{n}+a_{6} C_{i-2}^{n}+a_{5} C_{i-1}^{n} \text {. }
$$ 
Substituting (12) into (8) gives the following equation:

$$
\begin{aligned}
& 2 \sum_{j=i-3}^{i-1} C_{j}^{n+1} T B_{j}\left(x_{i}\right)-k \alpha^{2} \sum_{j=i-3}^{i-1} C_{j}^{n+1} T B_{j}^{\prime \prime}\left(x_{i}\right) \\
& =2 \sum_{j=i-3}^{i-1} C_{j}^{n} T B_{j}\left(x_{i}\right)+k \alpha^{2} \sum_{j=i-3}^{i-1} C_{j}^{n} T B_{j}^{\prime \prime}\left(x_{i}\right) \\
& \quad+2 k q\left(x_{i}, t_{n+1}\right) .
\end{aligned}
$$

The system thus obtained on simplifying (15) consists of $N+1$ linear equations in $N+3$ unknowns $C^{n+1}=$ $\left(C_{-3}^{n+1}, C_{-2}^{n+1}, C_{-1}^{n+1}, \ldots, C_{N-1}^{n+1}\right)$ at the time level $t=t_{n+1}$. Equation (9) is applied to the boundary constraints (2) and (3) for two additional linear equations to obtain a unique solution of the resulting system:

$$
\begin{aligned}
& \xi_{1} U\left(0, t_{n+1}\right)+\xi_{2} U_{x}\left(0, t_{n+1}\right) \\
&= \int_{0}^{L} g_{2}(x) u\left(x, t_{n+1}\right) d x+h_{1}\left(t_{n+1}\right), \\
& \xi_{3} U\left(L, t_{n+1}\right)+\xi_{4} U_{x}\left(L, t_{n+1}\right) \\
&=\int_{0}^{L} g_{3}(x) u\left(x, t_{n+1}\right) d x+h_{2}\left(t_{n+1}\right) .
\end{aligned}
$$

From (15), (16), and (17), the system can be written in the matrix vector form as follows:

$$
M C^{n+1}=N C^{n}+b
$$

where

$$
\begin{gathered}
C^{n+1}=\left[C_{-3}^{n+1}, C_{-2}^{n+1}, C_{-1}^{n+1}, \ldots, C_{N-1}^{n+1}\right]^{T}, \\
C^{n}=\left[C_{-3}^{n}, C_{-2}^{n}, C_{-1}^{n}, \ldots, C_{N-1}^{n}\right]^{T}, \\
b=\left[\alpha_{1}\left(t_{n+1}\right), 2 k q\left(x_{0}, t_{n+1}\right), 2 k q\left(x_{1}, t_{n+1}\right),\right. \\
\left.\ldots, 2 k q\left(x_{N}, t_{n+1}\right), \beta_{1}\left(t_{n+1}\right)\right]^{T}, \\
n=0,1,2, \ldots, M
\end{gathered}
$$

and $M$ and $N$ are $N+3$-dimensional matrix given by

$$
\begin{aligned}
M & =\left[\begin{array}{ccccccc}
p_{0} & q_{0} & p_{00} & 0 & \cdots & 0 & 0 \\
p_{1} & q_{1} & p_{1} & 0 & \cdots & 0 & 0 \\
0 & p_{1} & q_{1} & p_{1} & 0 & \cdots & 0 \\
\vdots & \vdots & \vdots & \vdots & \vdots & \vdots & \vdots \\
\vdots & \vdots & \vdots & \vdots & \vdots & \vdots & \vdots \\
\vdots & \vdots & \vdots & \vdots & \vdots & \vdots & \vdots \\
0 & 0 & \cdots & \cdots & p_{1} & q_{1} & p_{1} \\
0 & 0 & \cdots & \cdots & p_{0} & q_{0} & p_{00}
\end{array}\right], \\
N & =\left[\begin{array}{ccccccc}
0 & 0 & 0 & 0 & \cdots & 0 & 0 \\
p_{2} & q_{2} & p_{2} & 0 & \cdots & 0 & 0 \\
0 & p_{2} & q_{2} & p_{2} & 0 & \cdots & 0 \\
\vdots & \vdots & \vdots & \vdots & \vdots & \vdots & \vdots \\
0 & 0 & \cdots & \cdots & p_{2} & q_{2} & p_{2} \\
0 & 0 & \cdots & \cdots & 0 & 0 & 0
\end{array}\right],
\end{aligned}
$$

where

$$
\begin{gathered}
p_{0}=\xi_{1} a_{1}+\xi_{2} a_{3}, \quad q_{0}=\xi_{1} a_{1}, \\
p_{00}=\xi_{1} a_{1}+\xi_{2} a_{4}, \quad p_{1}=2 a_{1}-k \alpha^{2} a_{5}, \\
q_{1}=2 a_{2}-k \alpha^{2} a_{6}, \quad p_{2}=2 a_{1}+k \alpha^{2} a_{5}, \\
q_{2}=2 a_{2}+k \alpha^{2} a_{6}, \\
\alpha_{1}\left(t_{n+1}\right)=\int_{0}^{L} g_{2}(x) u\left(x, t_{n+1}\right) d x+h_{1}\left(t_{n+1}\right), \\
\beta_{1}\left(t_{n+1}\right)=\int_{0}^{L} g_{3}(x) u\left(x, t_{n+1}\right) d x+h_{2}\left(t_{n+1}\right) .
\end{gathered}
$$

Thus, the system (18) becomes a matrix system of dimension $(N+3) \times(N+3)$ which is a tridiagonal system that can be solved by the Thomas Algorithm [16].

3.1. Initial State Vector $C^{0}$. After the initial vectors $C^{0}$ have been computed from the initial constraints, the approximate solutions $U_{i}^{n+1}$ at a particular time level can be calculated repeatedly by solving the recurrence relation (15) [40].

The initial vectors $C^{0}$ can be obtained from the initial condition and boundary values of the derivatives of the initial condition as follows $[40,49]$ :

$$
\begin{gathered}
\left(U_{i}^{0}\right)_{x}=g_{1}^{\prime}\left(x_{i}\right), \quad i=0, \\
U_{i}^{0}=g_{1}\left(x_{i}\right), \quad i=0,1,2, \ldots, N, \\
\left(U_{i}^{0}\right)_{x}=g_{1}^{\prime}\left(x_{i}\right), \quad i=N .
\end{gathered}
$$

Thus (22) yields a $(N+3) \times(N+3)$ matrix system, of the form

$$
A C^{n}=d,
$$

where

$$
\begin{gathered}
C^{n}=\left[C_{-3}^{n}, C_{-2}^{n}, C_{-1}^{n}, \ldots, C_{N-1}^{n}\right]^{T}, \\
d=\left[g_{1}^{\prime}\left(x_{0}\right), g_{1}\left(x_{0}\right), \ldots, g_{1}\left(x_{N}\right), g_{1}^{\prime}\left(x_{N}\right)\right]^{T}, \quad n=0 \\
A=\left[\begin{array}{ccccccc}
a_{3} & 0 & a_{4} & 0 & \cdots & 0 & 0 \\
a_{1} & a_{2} & a_{1} & 0 & \cdots & 0 & 0 \\
0 & a_{1} & a_{2} & a_{1} & 0 & \cdots & 0 \\
\vdots & \vdots & \vdots & \vdots & \vdots & \vdots & \vdots \\
\vdots & \vdots & \vdots & \vdots & \vdots & \vdots & \vdots \\
\vdots & \vdots & \vdots & \vdots & \vdots & \vdots & \vdots \\
0 & 0 & \cdots & \cdots & a_{1} & a_{2} & a_{1} \\
0 & 0 & \cdots & \cdots & a_{3} & 0 & a_{4}
\end{array}\right]
\end{gathered}
$$

The solution of this system can be found by the use of the Thomas Algorithm.

\section{Stability}

In this section, the von Neumann stability method is applied for investigating the stability of the proposed scheme. This 
approach has been used by many researchers [18, 40, 44, $45,47-49]$. Substituting the approximate solution $U$ and its derivatives at knots with $q(x, t)=0$, into (6) yields a difference equation with variables $C_{m}$ given by

$$
\begin{aligned}
\left(a_{1}-\right. & \left.k \theta \alpha^{2} a_{5}\right) C_{m-3}^{n+1}+\left(a_{2}-k \theta \alpha^{2} a_{6}\right) C_{m-2}^{n+1} \\
& +\left(a_{1}-k \theta \alpha^{2} a_{5}\right) C_{m-1}^{n+1} \\
= & \left(a_{1}+k(1-\theta) \alpha^{2} a_{5}\right) C_{m-3}^{n}+\left(a_{2}+k(1-\theta) \alpha^{2} a_{6}\right) C_{m-2}^{n} \\
& +\left(a_{1}+k(1-\theta) \alpha^{2} a_{5}\right) C_{m-1}^{n} .
\end{aligned}
$$

Substituting the values of $a_{i}, i=1,2,5,6$ into (25) we obtain

$$
\begin{aligned}
\left(p_{1}-\right. & \left.k^{2} \theta \alpha^{2} p_{2}\right) C_{m-3}^{n+1}+\left(p_{3}+k^{2} \theta \alpha^{2} p_{4}\right) C_{m-2}^{n+1} \\
& +\left(p_{1}-k^{2} \theta \alpha^{2} p_{2}\right) C_{m-1}^{n+1} \\
= & \left(p_{1}+k^{2}(1-\theta) \alpha^{2} p_{2}\right) C_{m-3}^{n} \\
& +\left(p_{3}-k^{2}(1-\theta) \alpha^{2} p_{4}\right) C_{m-2}^{n} \\
& +\left(p_{1}+k^{2}(1-\theta) \alpha^{2} p_{2}\right) C_{m-1}^{n},
\end{aligned}
$$

where

$$
\begin{aligned}
p_{1}= & 16 \sin ^{2}\left(\frac{h}{2}\right)\left(2 \cos \left(\frac{h}{2}\right)+\cos \left(\frac{3 h}{2}\right)\right) \\
& \times\left(1+2 \cos \left(\frac{h}{2}\right)\right), \\
p_{2}= & 3 \sin (h) \sin \left(\frac{3 h}{2}\right)\left(1+3 \cos \left(\frac{h}{2}\right)\right) \cos e^{2}\left(\frac{h}{2}\right) \\
& \times\left(1+2 \cos \left(\frac{h}{2}\right)\right), \\
p_{3}= & 32 \sin (h) \sin \left(\frac{3 h}{2}\right)\left(2 \cos \left(\frac{h}{2}\right)+\cos \left(\frac{3 h}{2}\right)\right), \\
p_{4}= & 24 \cot ^{2}\left(\frac{h}{2}\right) \sin h \sin \left(\frac{3 h}{2}\right)\left(2 \cos \left(\frac{h}{2}\right)+\cos \left(\frac{3 h}{2}\right)\right) .
\end{aligned}
$$

Simplifying it leads to

$$
\begin{aligned}
& w_{1} C_{m-3}^{n+1}+w_{2} C_{m-2}^{n+1}+w_{1} C_{m-1}^{n+1} \\
& \quad=w_{3} C_{m-3}^{n}+w_{4} C_{m-2}^{n}+w_{3} C_{m-1}^{n}
\end{aligned}
$$

where

$$
\begin{aligned}
& w_{1}=\left(p_{1}-k \theta \alpha^{2} p_{2}\right), \\
& w_{2}=\left(p_{3}+k \theta \alpha^{2} p_{4}\right),
\end{aligned}
$$

$$
\begin{aligned}
& w_{3}=\left(p_{1}+k(1-\theta) \alpha^{2} p_{2}\right), \\
& w_{4}=\left(p_{3}-k(1-\theta) \alpha^{2} p_{4}\right) .
\end{aligned}
$$

Now on inserting the trial solutions (one Fourier mode out of the full solution) at a given point $x_{m}$ is $C_{m}^{n}=\delta^{n} \exp (i m \eta h)$ into (28) and rearranging the equations, $\eta$ is the mode number, $h$ is the element size, and $i=\sqrt{-1}$, we get

$$
\begin{aligned}
w_{1} \delta^{n+1} e^{i \eta(m-3) h}+w_{2} \delta^{n+1} e^{i \eta(m-2) h}+w_{1} \delta^{n+1} e^{i \eta(m-1) h} \\
=w_{3} \delta^{n} e^{i \eta(m-3) h}+w_{4} \delta^{n} e^{i \eta(m-2) h}+w_{3} \delta^{n} e^{i \eta(m-1) h} .
\end{aligned}
$$

Dividing (30) by $\delta^{n} e^{i \eta(m-2) h}$ and rearranging the equation, we get

$$
\delta\left(w_{2}+w_{1} \cos (\eta h)\right)=\left(w_{4}+w_{3} \cos (\eta h)\right) .
$$

Let

$$
\begin{aligned}
& A=p_{3}+p_{1} \cos (\eta h) \\
& B=k \alpha^{2}\left(p_{4}-p_{2} \cos (\eta h)\right) .
\end{aligned}
$$

Therefore, (31) can be written as

$$
\delta(A+\theta B)-(A-(1-\theta) B)=0 .
$$

Equation (33) can be rewritten as

$$
\delta\left[X_{2}+i Y\right]-\left[X_{1}+i Y\right]=0
$$

where

$$
\begin{gathered}
X_{1}=(A-(1-\theta) B), \\
X_{2}=(A+\theta B), \\
Y=0 .
\end{gathered}
$$

For stability, the maximum modulus of the eigenvalues of the matrix has to be less than or equal to one [47]. Since $A>0$, $B>0$, and $0 \leq \theta \leq 1$, we always have

$$
|\xi|^{2}=\frac{X_{1}^{2}+Y^{2}}{X_{2}^{2}+Y^{2}} \leq 1 .
$$

Thus, from (36), the proposed scheme for nonclassical diffusion equation (with term, $q(x, t)=0$ ) is unconditionally stable and it is also unconditionally stable with a general term $q(x, t)$, since the modulus of the eigenvalues must be less than one [47]. We recall Duhamel's principle ([15], chapter 9); a scheme is stable for equation $P_{k, h} v=f$ if it is stable for the equation $P_{k, h} v=0$. This means that there are no constraints on grid size $h$ and step size in time level $k$, but we should prefer those values of $h$ and $k$ for which we obtain the best accuracy of the scheme. 
TABLE 2: Absolute errors for Example 1 with $h=0.05$ and several values of time step by present method (CuTBSM) and compare with TMOL $[10]$.

\begin{tabular}{|c|c|c|c|c|c|c|}
\hline$t$ & $\begin{array}{c}k=0.01 \\
\text { TMOL [10] }\end{array}$ & Present method & $\begin{array}{c}k=0.005 \\
\text { TMOL [10] }\end{array}$ & Present method & $\begin{array}{c}k=0.001 \\
\text { TMOL [10] }\end{array}$ & Present method \\
\hline 0.1 & $4.5 E-04$ & $4.31 E-04$ & $4.0 E-05$ & $1.96 E-04$ & $1.5 E-05$ & $8.25 E-06$ \\
\hline 0.3 & $1.4 E-03$ & $5.88 E-04$ & $1.4 E-04$ & $2.69 E-04$ & $2.5 E-05$ & $1.33 E-05$ \\
\hline 0.5 & $2.5 E-03$ & $5.20 E-04$ & $2.5 E-04$ & $2.38 E-04$ & $2.4 E-05$ & $1.20 E-05$ \\
\hline 0.7 & $4.0 E-03$ & $4.23 E-04$ & $4.0 E-04$ & $1.92 E-04$ & $2.7 E-05$ & $8.36 E-06$ \\
\hline 0.9 & $5.5 E-03$ & $3.32 E-04$ & $5.5 E-04$ & $1.50 E-04$ & $6.0 E-05$ & $4.03 E-06$ \\
\hline 1.0 & $6.0 E-04$ & $2.91 E-04$ & $6.0 E-04$ & $1.31 E-04$ & $6.8 E-05$ & $1.90 E-06$ \\
\hline
\end{tabular}

\section{Results and Discussions}

In this section, the cubic trigonometric B-spline collocation method is employed to obtain the numerical solutions for one-dimensional nonclassical diffusion problem with nonlocal boundary constraints given in (1)-(3). Two numerical examples are discussed in this section to exhibit the capability and efficiency of the proposed trigonometric spline method. Numerical results are compared with existing methods in the literature and with the exact solution at the different nodal points $x_{i}$ for some time levels $t_{n}$ using some particular space step size $h$ and time step $k$. In order to calculate the maximum errors and relative $L_{2}$ error norms of the proposed method numerically, we use the following formulas:

$$
\begin{gathered}
L_{\infty}=\max _{i}\left|U_{\text {num }}\left(x_{i}, T\right)-u_{\text {exact }}\left(x_{i}, T\right)\right|, \\
L_{2}=\frac{\left|U_{\text {num }}(x, t)-u_{\text {exact }}(x, t)\right|}{\left|u_{\text {exact }}(x, t)\right|} .
\end{gathered}
$$

Example 1. Consider the nonclassical diffusion problem ((1)(3)), with

$$
\begin{gathered}
q(x, t)=-e^{-(x+\sin t)}(1+\cos t), \quad 0 \leq x \leq 1, \quad 0<t \leq 1, \\
g_{1}(x)=e^{-x}, \quad 0 \leq x \leq 1, \\
\xi_{i}(i=1,3)=1, \quad \xi_{i}(i=2,4)=0, \\
g_{2}(x)=\frac{e}{e-2} x, \quad g_{3}(x)=\frac{2}{e+\sin 1-\cos 1} \cos x, \\
L=1, \\
h_{i}(t)(i=1,2)=0, \quad 0<t \leq 1 .
\end{gathered}
$$

This test problem is from Dehghan [8] and $\mathrm{Li}$ and $\mathrm{Wu}$ [10] and the known solution is $u(x, t)=e^{-(x+\sin t)}$. We compare the maximum errors with TMOL [10] when they are considered with space size $h=0.05$ and errors are recorded with several values of time step $k$, given in Table 2 and also shown in Figure 1. It is worth noting that the results obtained using CuTBSM are more accurate as compared to TMOL [10]. We also compare the relative errors of numerical value of $u(0.6,0.1)$ with different space step $h=0.05,0.025,0.01$

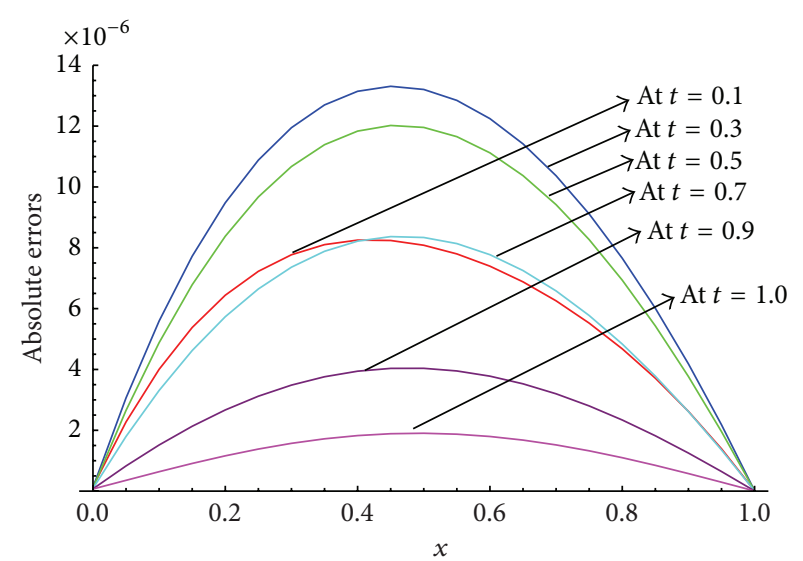

FIGURE 1: Absolute errors of numerical values at different time levels for Example 1 by using $h=0.05$ and $k=0.001$.

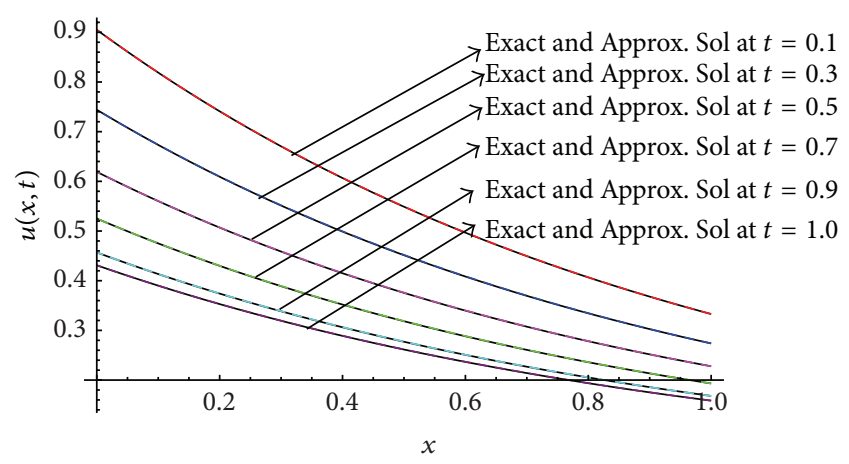

Figure 2: A comparison of numerical solution and known solution at different time levels for Example 1 with $h=0.05$ and $k=0.01$.

and with time step size $k=s h^{2}, s=1,2,4,5$, instead of $k=0.4 h^{2}$ which was used in the BTCS [8], the implicit $(3,3)$ Crandall's formula [8], the 3-point FTCS [8], and the Dufort-Frankel three-level approach [8] and TMOL [10], and these results are tabulated in Table 3. It is clearly shown from this table that the obtained results by using CuTBSM are more precise as compared to methods in $[8,10]$. Figure 2 shows the approximate solution and exact solution for this example at different time levels with $h=0.05$ and $k=0.01$. 
TABLE 3: Relative errors of numerical method at $u(0.6,0.1)$ for Example 1 with $k=0.4 h^{2}$ and various values of space step.

\begin{tabular}{|c|c|c|c|c|c|}
\hline$h$ & $\begin{array}{l}\text { Present method } \\
\qquad\left(k=5 h^{2}\right)\end{array}$ & $\begin{array}{l}\text { Present method } \\
\qquad\left(k=4 h^{2}\right)\end{array}$ & $\begin{array}{l}\text { Present method } \\
\quad\left(k=2 h^{2}\right)\end{array}$ & $\begin{array}{l}\text { Present method } \\
\left(k=h^{2}\right)\end{array}$ & $\begin{array}{l}\text { Present method } \\
\quad\left(k=0.4 h^{2}\right)\end{array}$ \\
\hline 0.05 & $4.94 E-04$ & $3.88 E-04$ & $1.76 E-04$ & $7.08 E-05$ & $7.39 E-06$ \\
\hline 0.025 & $1.23 E-04$ & $9.69 E-05$ & $4.41 E-05$ & $1.77 E-05$ & $1.87 E-06$ \\
\hline 0.010 & $1.97 E-05$ & $1.55 E-05$ & $7.08 E-06$ & $2.86 E-06$ & $3.26 E-07$ \\
\hline$h$ & $\begin{array}{l}\text { BTCS }[8] \\
\left(k=0.4 h^{2}\right)\end{array}$ & $\begin{array}{l}\text { Crandall [8] } \\
\left(k=0.4 h^{2}\right)\end{array}$ & $\begin{array}{c}\text { FTCS }[8] \\
\left(k=0.4 h^{2}\right)\end{array}$ & $\begin{array}{c}\text { Dufort-Frankel } \\
{[8]} \\
\left(k=0.4 h^{2}\right)\end{array}$ & $\begin{array}{l}\text { TMOL }[10] \\
\left(k=0.4 h^{2}\right)\end{array}$ \\
\hline 0.05 & $6.3 E-02$ & $3.9 E-03$ & $6.4 E-02$ & $6.8 E-02$ & $2.8 E-04$ \\
\hline 0.025 & $1.5 E-02$ & $2.4 E-04$ & $1.6 E-02$ & $1.7 E-02$ & $7.1 E-05$ \\
\hline 0.010 & $4.0 E-03$ & $1.5 E-05$ & $4.1 E-03$ & $4.1 E-03$ & - \\
\hline
\end{tabular}

TABLE 4: Absolute errors for Example 2 with $h=0.05$ and several values of time step by present method and TMOL [10].

\begin{tabular}{|c|c|c|c|c|c|c|c|c|}
\hline$t$ & $\begin{array}{c}k=0.01 \\
\text { TMOL [10] }\end{array}$ & Present method & $\begin{array}{c}k=0.005 \\
\text { TMOL [10] }\end{array}$ & Present method & $\begin{array}{c}k=0.001 \\
\text { TMOL [10] }\end{array}$ & Present method & $\begin{array}{l}k=0.0005 \\
\text { TMOL [10] }\end{array}$ & Present method \\
\hline 0.1 & $7.0 E-04$ & $1.80 E-03$ & $1.6 E-04$ & $8.66 E-04$ & $8.0 E-05$ & $1.15 E-04$ & $4.0 E-05$ & $2.31 E-05$ \\
\hline 0.3 & $9.0 E-04$ & $1.77 E-03$ & $1.8 E-04$ & $8.44 E-04$ & $1.0 E-05$ & $9.95 E-05$ & $5.0 E-05$ & $8.09 E-06$ \\
\hline 0.5 & $7.0 E-04$ & $1.16 E-03$ & $1.4 E-04$ & $5.48 E-04$ & $7.0 E-05$ & $5.49 E-05$ & $3.5 E-05$ & $8.27 E-06$ \\
\hline 0.7 & $4.5 E-04$ & $7.56 E-04$ & $9.0 E-05$ & $3.51 E-04$ & $4.5 E-05$ & $2.79 E-05$ & $2.4 E-05$ & $1.31 E-06$ \\
\hline 0.9 & $3.0 E-04$ & $5.09 E-04$ & $6.0 E-05$ & $2.33 E-04$ & $3.0 E-05$ & $1.33 E-05$ & $1.4 E-05$ & $1.46 E-06$ \\
\hline 1.0 & $2.4 E-04$ & $4.25 E-04$ & $4.5 E-05$ & $1.93 E-04$ & $2.4 E-05$ & $8.84 E-06$ & $1.2 E-05$ & $1.46 E-06$ \\
\hline
\end{tabular}

TABLE 5: Absolute errors of numerical value of $u$ for Example 2 with different space step size, $k=0.0001$, at selected time levels and compare with CSCM [11].

\begin{tabular}{lcccccc}
\hline$t$ & $h=0.25$ & Present method & $\begin{array}{c}h=0.125 \\
\text { CSCM [11] }\end{array}$ & Present method & $\begin{array}{c}h=0.0625 \\
\text { CSCM [11] }\end{array}$ & Present method \\
\hline 0.1 & $1.07 E-02$ & $1.91 E-03$ & $1.80 E-03$ & $4.50 E-04$ & $3.22 E-04$ & $9.77 E-05$ \\
0.5 & $8.60 E-03$ & $1.73 E-03$ & $1.60 E-03$ & $4.21 E-04$ & $2.77 E-04$ & $9.58 E-05$ \\
0.6 & $7.90 E-03$ & $1.52 E-03$ & $1.40 E-03$ & $3.70 E-04$ & $2.53 E-04$ & $8.49 E-05$ \\
0.9 & $5.70 E-03$ & $1.06 E-03$ & $1.00 E-03$ & $2.58 E-04$ & $1.84 E-04$ & $6.04 E-05$ \\
1.0 & $5.10 E-03$ & $9.50 E-04$ & $9.26 E-04$ & $2.32 E-04$ & $1.64 E-04$ & $5.44 E-05$ \\
\hline
\end{tabular}

Example 2. We consider another numerical test problem, with

$$
\begin{gathered}
q(x, t)=\frac{-2\left(x^{2}+t+1\right)}{(t+1)^{3}} \quad 0 \leq x \leq 1, \quad 0<t \leq 1, \\
g_{1}(x)=x^{2}, \quad 0 \leq x \leq 1, \\
\xi_{i}(i=1,3)=1, \quad \xi_{i}(i=2,4)=0, \quad g_{2}(x)=x, \\
g_{3}(x)=x, \quad L=1, \quad 0 \leq x \leq 1, \\
h_{1}(t)=\frac{-1}{4(t+1)^{2}}, \\
h_{2}(t)=\frac{3}{4(t+1)^{2}} \\
0<t \leq 1 .
\end{gathered}
$$

The exact solution of this equation is $u(x, t)=x^{2} /(t+1)^{2}$ and this test problem has been taken from $[8,10,11]$. This problem is tested using different values of $h$ and $k$ to show the capability of the present method for solving nonclassical diffusion equation $((1)-(3))$. The final time is taken $T=1.0$. The maximum errors of the numerical method are calculated at different time levels with different time step size and it is observed that they are more accurate as compared to TMOL [10] and Chebyshev spectral collocation method (CSCM) based on Chebyshev polynomials [11]. The numerical errors are tabulated in Tables 4 and 5 and are also depicted graphically in Figure 3. The relative errors of numerical value $u(0.6,1.0)$ with different space step $h=0.05,0.025,0.01$ and with time step size $k=s h^{2}, s=1,2,4,5$, instead of $k=$ $0.4 h^{2}$ which was used in the BTCS $[8]$, the implicit $(3,3)$ Crandall's formula [8], the 3-point FTCS [8], and the DufortFrankel three-level approach [8] and TMOL [10] and they are recorded in Table 6 . It is worth noting that numerical results are much better than the methods in $[8,10,11]$. A comparison 
TABLE 6: Relative errors of numerical method at $u(0.6,1.0)$ for Example 2 with various values of space step.

\begin{tabular}{lccccc}
\hline$h$ & $\begin{array}{c}\text { Present method } \\
\left(k=5 h^{2}\right)\end{array}$ & $\begin{array}{c}\text { Present method } \\
\left(k=4 h^{2}\right)\end{array}$ & $\begin{array}{c}\text { Present method } \\
\left(k=2 h^{2}\right)\end{array}$ & $\begin{array}{c}\text { Present method } \\
\left(k=h^{2}\right)\end{array}$ & $\begin{array}{c}\text { Present method } \\
\left(k=0.4 h^{2}\right)\end{array}$ \\
\hline 0.05 & $5.28 E-04$ & $4.15 E-04$ & $1.89 E-04$ & $7.81 E-05$ & $8.84 E-06$ \\
0.025 & $1.14 E-04$ & $8.96 E-05$ & $4.74 E-05$ & $1.91 E-05$ & $3.21 E-06$ \\
0.010 & $2.11 E-05$ & $1.66 E-05$ & $7.59 E-06$ & $3.06 E-06$ & TMOL [10] \\
\hline$h$ & BTCS [8] & Crandall [8] & FTCS [8] & Dufort-Frankel [8] & $\left(k=0.4 h^{2}\right)$ \\
\hline 0.05 & $\left(k=0.4 h^{2}\right)$ & $\left(k=0.4 h^{2}\right)$ & $\left(k=0.4 h^{2}\right)$ & $7.8 E-02$ & $1.6 E-03$ \\
0.025 & $7.3 E-02$ & $3.8 E-03$ & $7.5 E-02$ & $1.9 E-02$ & $4.0 E-04$ \\
0.010 & $1.8 E-02$ & $2.1 E-04$ & $1.9 E-02$ & $3.9 E-03$ & - \\
\hline
\end{tabular}

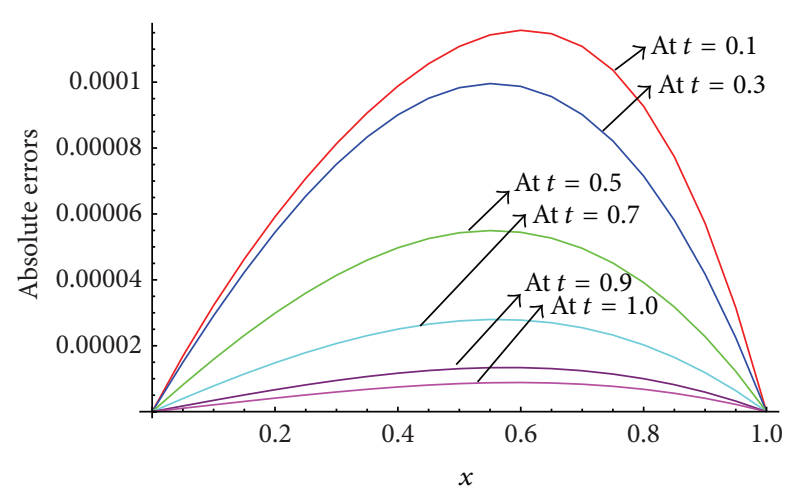

FIGURE 3: Absolute errors of numerical values at different time levels for Example 2 by using $h=0.05$ and $k=0.001$.

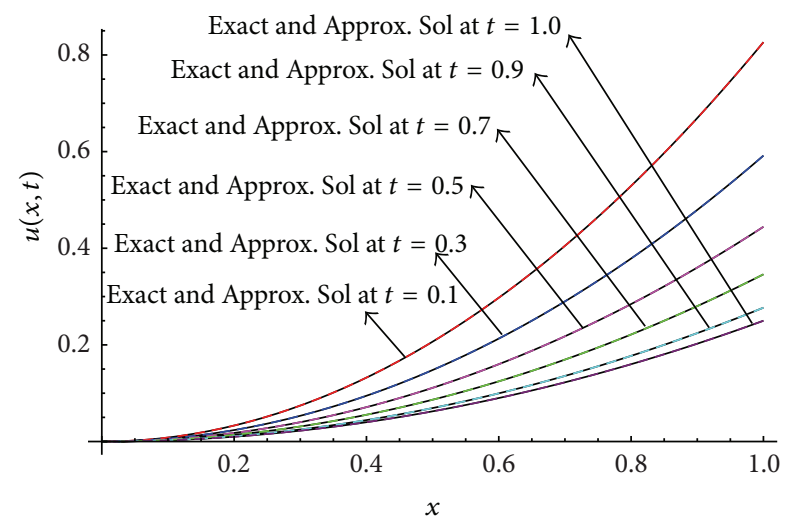

FIGURE 4: A comparison of numerical solution and known solution at different time levels for Example 2 with $h=0.05$ and $k=0.01$.

of numerical solutions at different time levels with known solution is presented graphically in the Figure 4.

Example 3. Finally, we consider nonclassical diffusion problem ((1)-(3)), with

$$
\begin{gathered}
q(x, t)=\frac{-e^{x}(1+t)^{2}}{\left(1+t^{2}\right)^{2}}, \quad 0 \leq x \leq 1,0<t \leq 1, \\
g_{1}(x)=e^{x}, \quad 0 \leq x \leq 1,
\end{gathered}
$$

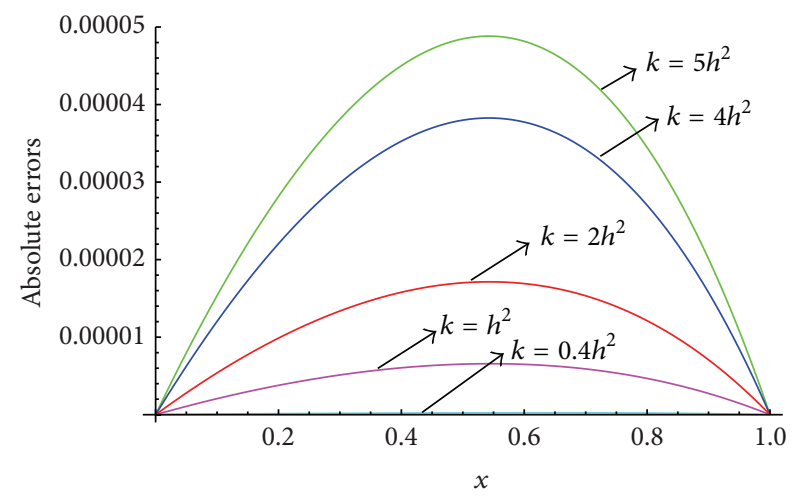

Figure 5: Absolute errors of numerical values at $T=1$ for Example 3 with $h=0.01$ and different time step size.

$$
\begin{gathered}
\xi_{i}(i=1,3)=1, \quad \xi_{i}(i=2,4)=0, \quad g_{2}(x)=\frac{(x+1)}{e}, \\
g_{3}(x)=0, \quad L=1, \quad 0 \leq x \leq 1, \\
h_{1}(t)=0, \quad h_{2}(t)=\frac{e}{\left(1+t^{2}\right)} \quad 0<t \leq 1 .
\end{gathered}
$$

This test problem has been taken from [15] and its exact solution is $u(x, t)=e^{x} /\left(1+t^{2}\right)$. The final time is taken as $T=1$. The maximum errors of the proposed scheme are considered at $T=1$ with different time step sizes that are depicted graphically in Figure 5. The relative errors of numerical value $u(0.6,1.0)$ are calculated with different space size step with $k=s h^{2}, s=1,2,4,5$, and they are given in Table 7 . It is worth noting that the numerical results are found to be in good agreement with exact solutions. A comparison of numerical solutions at different time levels with known solution is presented graphically in Figure 6.

\section{Concluding Remarks}

In this paper, a new two-time level implicit scheme based on cubic trigonometric B-spline has been used to solve the nonclassical diffusion problem with known initial and with nonlocal boundary constraints instead of the usual boundary 
TABLE 7: Relative errors of numerical method at $u(0.6,1.0)$ for Example 3 with different time step size and various values of space step.

\begin{tabular}{lccccc}
\hline$h$ & $\begin{array}{c}\text { Present method } \\
\left(k=5 h^{2}\right)\end{array}$ & $\begin{array}{c}\text { Present method } \\
\left(k=4 h^{2}\right)\end{array}$ & $\begin{array}{c}\text { Present method } \\
\left(k=2 h^{2}\right)\end{array}$ & $\begin{array}{c}\text { Present method } \\
\left(k=h^{2}\right)\end{array}$ & $\begin{array}{c}\text { Present method } \\
\left(k=0.4 h^{2}\right)\end{array}$ \\
\hline 0.05 & $1.21 E-03$ & $9.45 E-04$ & $4.23 E-04$ & $1.62 E-04$ & $6.07 E-06$ \\
0.025 & $3.01 E-04$ & $2.35 E-04$ & $1.05 E-04$ & $4.05 E-05$ & $1.51 E-06$ \\
0.010 & $4.81 E-05$ & $3.77 E-05$ & $1.69 E-05$ & $6.49 E-06$ & $2.41 E-07$ \\
\hline
\end{tabular}

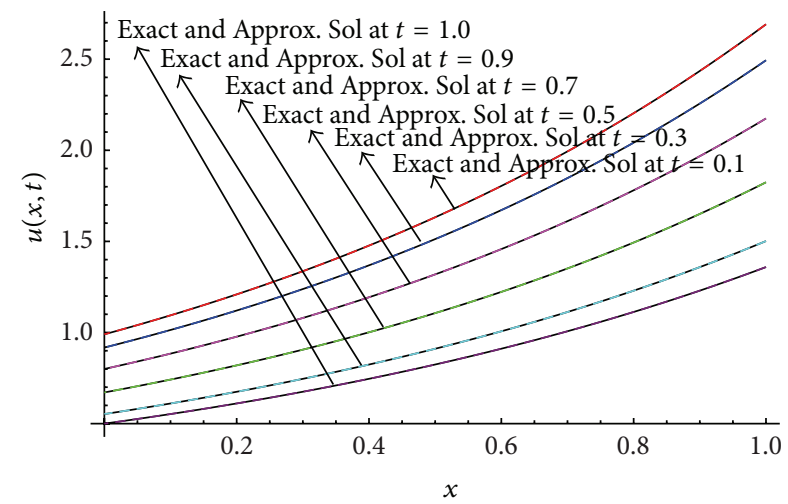

FIGURE 6: A comparison of numerical solution and known solution at different time levels for Example 3 with $h=0.05$ and $k=0.01$.

constraints. A usual finite difference discretization is used for time derivatives and cubic trigonometric B-spline is applied for space derivatives. It is noted that the accuracy of solution may reduce as time increases due to the time truncation errors of time derivative term [47]. The cubic trigonometric B-spline method used in this paper is simple and straightforward to apply. An advantage of using the cubic trigonometric B-spline method outlined in this paper is that it produces a spline function on each new time line which can be used to obtain the solutions at any intermediate point in the space direction whereas the finite difference approach yields the solution only at the selected points. The CuTBSM has approximated the solution with more accurate results for time step size $k=s h^{2}, s=1,2,4,5$, as compared to some finite difference schemes with smaller time step size $k=$ $0.4 h^{2}$ such as BTCS, Crandall's formula, FTCS, the DufortFrankel scheme, and TMOL based on reproducing kernel. The proposed method is shown to be unconditionally stable. It is also evident from the examples that the approximate solution is very close to the exact solution.

\section{Conflict of Interests}

The authors declare that there is no conflict of interests regarding the publication of this paper.

\section{Acknowledgments}

The authors are grateful to the anonymous reviewers for their helpful, valuable comments, and suggestions to improve the paper. This study was fully supported by FRGS Grant no. 203/PMATHS/6711324 from the School of Mathematical Sciences, Universiti Sains Malaysia, Penang, Malaysia. The first author was supported by Post Doctorate Fellowship from School of Mathematical Sciences, Universiti Sains Malaysia, Penang, Malaysia, during a part of the time in which the research was carried out.

\section{References}

[1] Y. S. Choi and K.-Y. Chan, "A parabolic equation with nonlocal boundary conditions arising from electrochemistry," Nonlinear Analysis: Theory, Methods \& Applications, vol. 18, no. 4, pp. 317331, 1992.

[2] A. Bouziani, "On a class of parabolic equations with a nonlocal boundary condition," Académie Royale de Belgique. Bulletin de la Classe des Sciences. 6e Série, vol. 10, no. 1-6, pp. 61-77, 1999.

[3] W. A. Day, "Parabolic equations and thermodynamics," Quarterly of Applied Mathematics, vol. 50, no. 3, pp. 523-533, 1992.

[4] A. A. Samarskiǔ, "Some problems of the theory of differential equations," Differential Equations, vol. 16, no. 11, pp. 1925-1935, 1980.

[5] A. R. Bahadır, "Application of cubic B-spline finite element technique to the termistor problem," Applied Mathematics and Computation, vol. 149, no. 2, pp. 379-387, 2004.

[6] J. H. Cushman, B. X. Hu, and F. Deng, "Nonlocal reactive transport with physical and chemical heterogeneity: localization errors," Water Resources Research, vol. 31, no. 9, pp. 22192237, 1995.

[7] F. Kanca, "The inverse problem of the heat equation with periodic boundary and integral overdetermination conditions," Journal of Inequalities and Applications, vol. 2013, article 108, 2013.

[8] M. Dehghan, "Efficient techniques for the second-order parabolic equation subject to nonlocal specifications," Applied Numerical Mathematics, vol. 52, no. 1, pp. 39-62, 2005.

[9] J. Martín-Vaquero and J. Vigo-Aguiar, "A note on efficient techniques for the second-order parabolic equation subject to non-local conditions," Applied Numerical Mathematics, vol. 59, no. 6, pp. 1258-1264, 2009.

[10] X. Li and B. Wu, "New algorithm for nonclassical parabolic problems based on the reproducing kernel method," Mathematical Sciences, vol. 7, article 4, 2013.

[11] A. Golbabai and M. Javidi, "A numerical solution for nonclassical parabolic problem based on Chebyshev spectral collocation method," Applied Mathematics and Computation, vol. 190, no. 1, pp. 179-185, 2007.

[12] W. A. Day, "A decreasing property of solutions of parabolic equations with applications to thermoelasticity;" Quarterly of Applied Mathematics, vol. 40, no. 4, pp. 468-475, 1983. 
[13] W. A. Day, "Extensions of a property of the heat equation to linear thermoelasticity and other theories," Quarterly of Applied Mathematics, vol. 40, no. 3, pp. 319-330, 1982.

[14] W. A. Day, Heat Conduction within Linear Thermoelasticity, vol. 30 of Springer Tracts in Natural Philosophy, Springer, New York, NY, USA, 1985.

[15] J. C. Strikwerda, Finite Difference Schemes and Partial Differential Equations, Society for Industrial and Applied Mathematics, Philadelphia, Pa, USA, 2nd edition, 2004.

[16] Du. V. Rosenberg, Methods for Solution of Partial Differential Equations, vol. 113, American Elsevier, New York, NY, USA, 1969.

[17] W. T. Ang, "A method of solution for the one-dimensional heat equation subject to nonlocal conditions," Southeast Asian Bulletin of Mathematics, vol. 26, no. 2, pp. 197-203, 2002.

[18] J. Martín-Vaquero and J. Vigo-Aguiar, "On the numerical solution of the heat conduction equations subject to nonlocal conditions," Applied Numerical Mathematics, vol. 59, no. 10, pp. 2507-2514, 2009.

[19] M. Dehghan, "On the numerical solution of the diffusion equation with a nonlocal boundary condition," Mathematical Problems in Engineering, vol. 2, pp. 81-92, 2003.

[20] M. Dehghan, "A computational study of the one-dimensional parabolic equation subject to nonclassical boundary specifications," Numerical Methods for Partial Differential Equations, vol. 22, no. 1, pp. 220-257, 2006.

[21] M. Tatari and M. Dehghan, "On the solution of the nonlocal parabolic partial differential equations via radial basis functions," Applied Mathematical Modelling, vol. 33, no. 3, pp. 1729-1738, 2009.

[22] J. H. Cushman, "Diffusion in fractal porous media," Water Resources Research, vol. 27, no. 4, pp. 643-644, 1991.

[23] V. V. Shelukhin, "A non-local in time model for radionuclides propagation in Stokes fluid," Siberian Branch of Russian Academy of Sciences, Institute of Hydrodynamics, no. 107, pp. 180-193, 1993.

[24] A. S. Vasudeva Murthy and J. G. Verwer, "Solving parabolic integro-differential equations by an explicit integration method," Journal of Computational and Applied Mathematics, vol. 39, no. 1, pp. 121-132, 1992.

[25] C. V. Pao, "Numerical methods for nonlinear integro-parabolic equations of Fredholm type," Computers \& Mathematics with Applications, vol. 41, no. 7-8, pp. 857-877, 2001.

[26] C. V. Pao, "Reaction diffusion equations with nonlocal boundary and nonlocal initial conditions," Journal of Mathematical Analysis and Applications, vol. 195, no. 3, pp. 702-718, 1995.

[27] J. R. Cannon and A. L. Matheson, "A numerical procedure for diffusion subject to the specification of mass," International Journal of Engineering Science, vol. 31, no. 3, pp. 347-355, 1993.

[28] M. Dehghan, "Numerical solution of a parabolic equation with non-local boundary specifications," Applied Mathematics and Computation, vol. 145, no. 1, pp. 185-194, 2003.

[29] G. Ekolin, "Finite difference methods for a nonlocal boundary value problem for the heat equation," BIT. Numerical Mathematics, vol. 31, no. 2, pp. 245-261, 1991.

[30] G. Fairweather and J. C. López-Marcos, "Galerkin methods for a semilinear parabolic problem with nonlocal boundary conditions," Advances in Computational Mathematics, vol. 6, no. 3-4, pp. 243-262, 1996.

[31] Y. Liu, "Numerical solution of the heat equation with nonlocal boundary conditions," Journal of Computational and Applied Mathematics, vol. 110, no. 1, pp. 115-127, 1999.
[32] G. E. Farin, Curves and Surfaces for Computer-Aided Geometric Design: A Practical Code, Academic Press, 1996.

[33] J. Hoschek and D. Lasser, Fundamentals of Computer Aided Geometric Design, A K Peters, Boston, Mass, USA, 1993.

[34] Y. S. Lai, W. P. Du, and R. H. Wang, "The viro method for construction of piecewise algebraic hypersurfaces," Abstract and Applied Analysis, vol. 2013, Article ID 690341, 7 pages, 2013.

[35] R. H. Wang, X. Q. Shi, Z. X. Luo, and Z. X. Su, Multivariate Spline and Its Applications, Science Press, Beijing, China; Kluwer Academic Publishers, New York, NY, USA, 2001.

[36] P. M. Prenter, Splines and Variational Methods, John Wiley \& Sons, New York, NY, USA, 1989.

[37] C. de Boor, A Practical Guide to Splines, vol. 27 of Applied Mathematical Sciences, Springer, 1978.

[38] H. N. Caglar, S. H. Caglar, and E. H. Twizell, "The numerical solution of third-order boundary-value problems with fourthdegree B-spline functions," International Journal of Computer Mathematics, vol. 71, no. 3, pp. 373-381, 1999.

[39] H. N. Çaglar, S. H. Çaglar, and E. H. Twizell, "The numerical solution of fifth-order boundary value problems with sixthdegree B-spline functions," Applied Mathematics Letters, vol. 12, no. 5, pp. 25-30, 1999.

[40] I. Dağ, D. Irk, and B. Saka, "A numerical solution of the Burgers' equation using cubic B-splines," Applied Mathematics and Computation, vol. 163, no. 1, pp. 199-211, 2005.

[41] J. Rashidinia, M. Ghasemi, and R. Jalilian, "A collocation method for the solution of nonlinear one-dimensional parabolic equations," Mathematical Sciences Quarterly Journal, vol. 4, no. 1, pp. 87-104, 2010.

[42] K. Qu, Z. Wang, and B. Jiang, "A finite element method by using bivariate splines for one dimensional heat equations," Journal of Information \& Computational Science, vol. 10, no. 12, pp. 3659$3666,2013$.

[43] J. Goh, A. A. Majid, and A. I. M. Ismail, "Numerical method using cubic B-spline for the heat and wave equation," Computers and Mathematics with Applications, vol. 62, no. 12, pp. 44924498, 2011.

[44] J. Goh, A. A. Majid, and A. I. M. Ismail, "Cubic B-spline collocation method for one-dimensional heat and advection-diffusion equations," Journal of Applied Mathematics, vol. 2012, Article ID 458701, 8 pages, 2012.

[45] J. Goh, A. A. Majid, and A. I. M. Ismail, "A comparison of some splines-based methods for the one-dimensional heat equation," Proceedings of World Academy of Science, Engineering and Technology, vol. 70, pp. 858-861, 2010.

[46] R. C. Mittal and G. Arora, "Quintic B-spline collocation method for numerical solution of the Kuramoto-Sivashinsky equation," Communications in Nonlinear Science and Numerical Simulation, vol. 15, no. 10, pp. 2798-2808, 2010.

[47] R. C. Mittal and G. Arora, "Numerical solution of the coupled viscous Burgers' equation," Communications in Nonlinear Science and Numerical Simulation, vol. 16, no. 3, pp. 1304-1313, 2011.

[48] M. Abbas, A. A. Majid, A. I. M. Ismail, and A. Rashid, "Numerical method using cubic B-spline for a strongly coupled reaction-diffusion system," PLOS ONE, vol. 9, no. 1, article e83265, 2014.

[49] M. Abbas, A. A. Majid, A. I. M. Ismail, and A. Rashid, "The application of cubic trigonometric B-spline to the numerical solution of the hyperbolic problems," Applied Mathematics and Computation, vol. 239, pp. 74-88, 2014. 
[50] A. Nikolis, "Numerical solutions of ordinary differential equations with quadratic trigonometric splines," Applied Mathematics E-Notes, vol. 4, pp. 142-149, 1995.

[51] N. N. Abd Hamid, A. A. Majid, and A. I. M. Ismail, "Cubic trigonometric B-spline applied to linear two-point boundary value problems of order two," World Academy of Science, Engineering and Technology, vol. 70, pp. 798-803, 2010. 


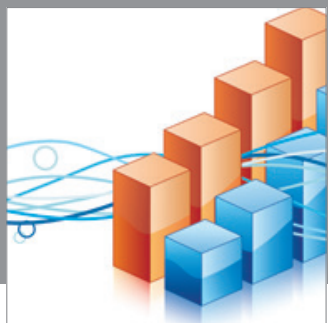

Advances in

Operations Research

mansans

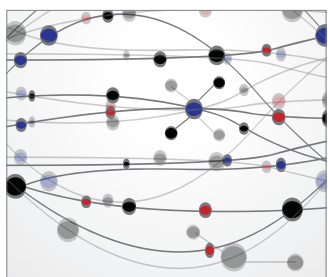

The Scientific World Journal
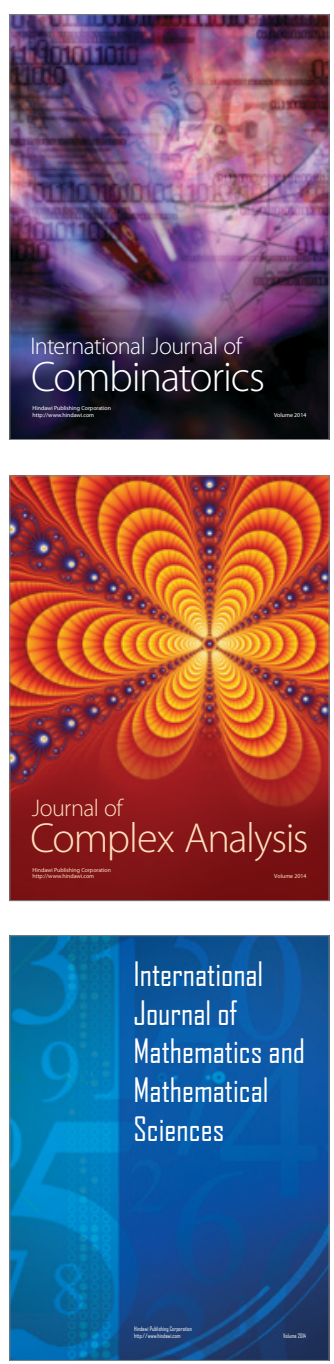
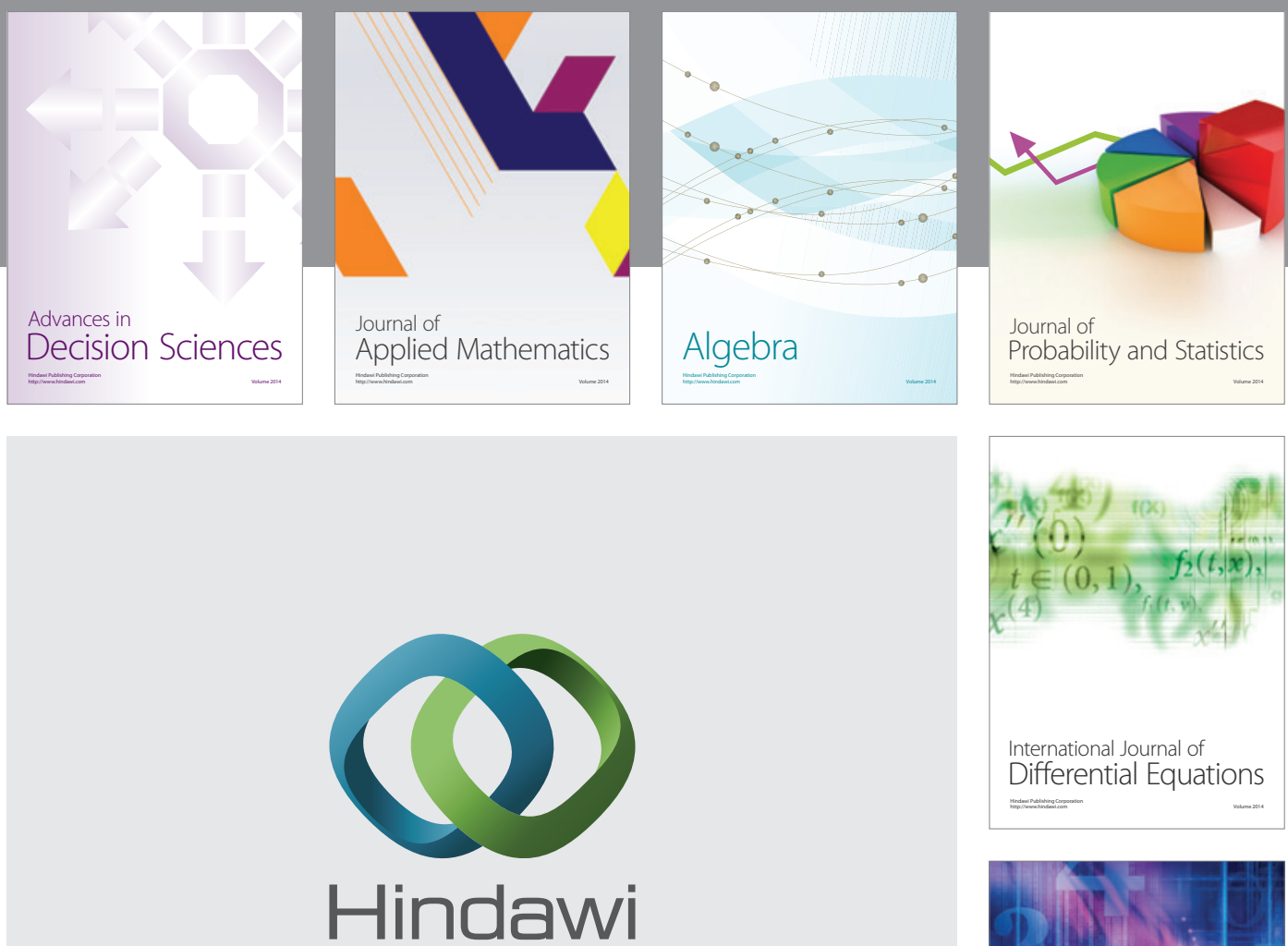

Submit your manuscripts at http://www.hindawi.com
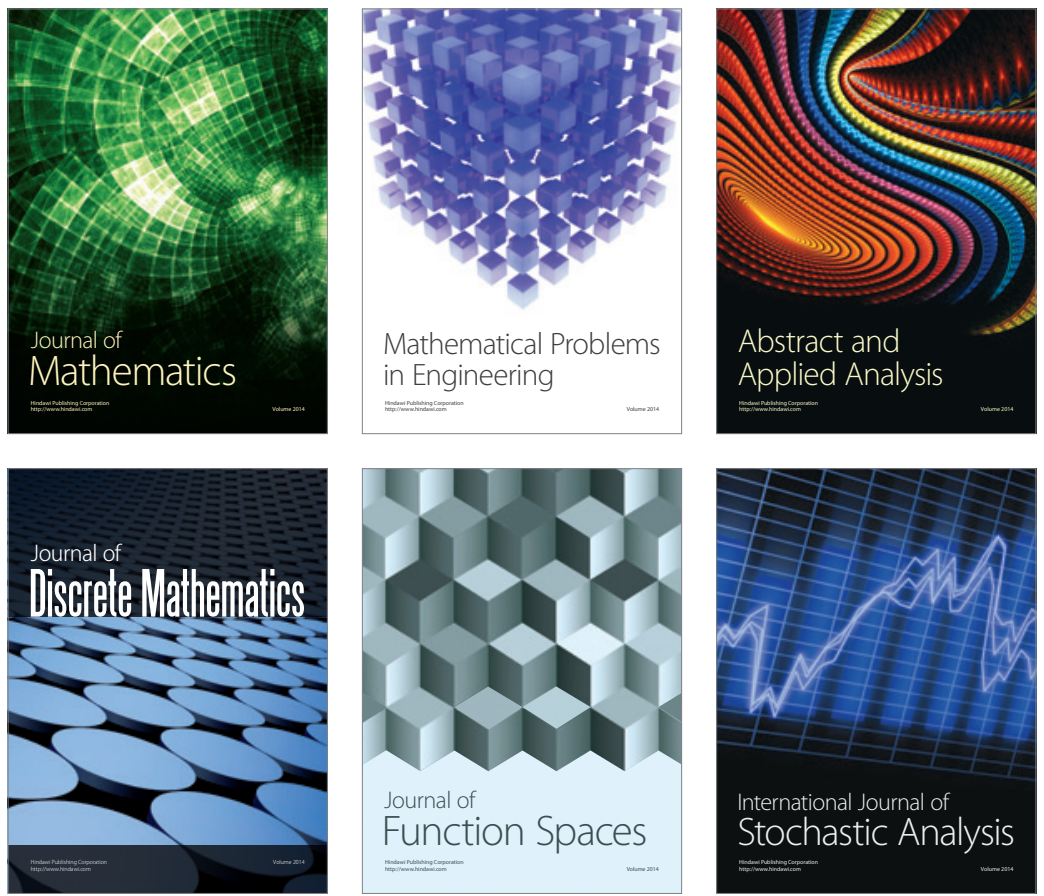

Journal of

Function Spaces

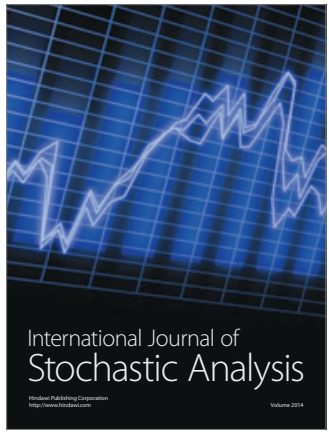

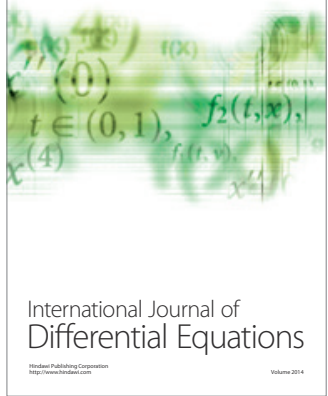
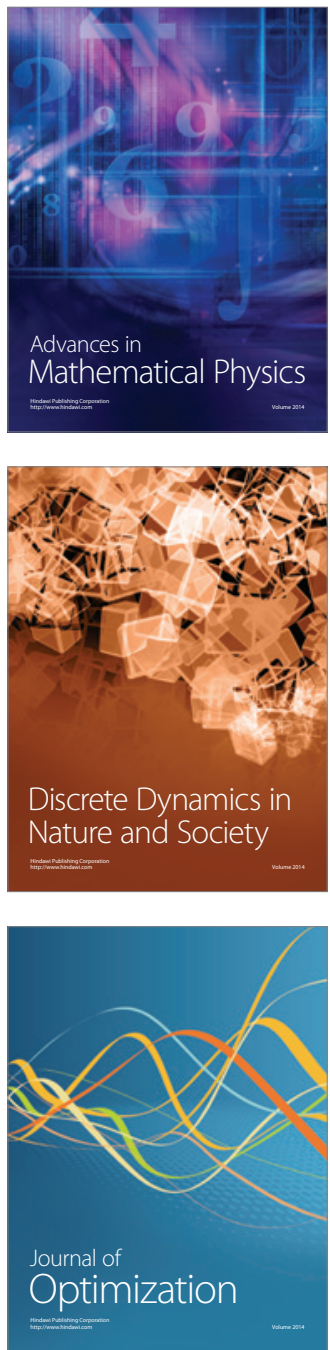